NATIONAL LABORATORY

\title{
Demonstration of Emitted-Neutron Computed Tomography to Quantify Nuclear Materials
}

September 2011

Prepared by

P. A. Hausladen, M. A. Blackston, and J. Newby

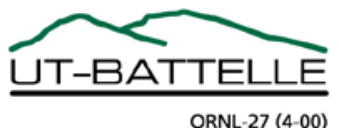




\section{DOCUMENT AVAILABILITY}

Reports produced after January 1, 1996, are generally available free via the U.S. Department of Energy (DOE) Information Bridge.

Web site http://www.osti.gov/bridge

Reports produced before January 1, 1996, may be purchased by members of the public from the following source.

National Technical Information Service

5285 Port Royal Road

Springfield, VA 22161

Telephone 703-605-6000 (1-800-553-6847)

TDD 703-487-4639

Fax 703-605-6900

E-mail info@ntis.gov

Web site http://www.ntis.gov/support/ordernowabout.htm

Reports are available to DOE employees, DOE contractors, Energy Technology Data Exchange (ETDE) representatives, and International Nuclear Information System (INIS) representatives from the following source.

Office of Scientific and Technical Information

P.O. Box 62

Oak Ridge, TN 37831

Telephone 865-576-8401

Fax 865-576-5728

E-mail reports@osti.gov

Web site http://www.osti.gov/contact.html

This report was prepared as an account of work sponsored by an agency of the United States Government. Neither the United States Government nor any agency thereof, nor any of their employees, makes any warranty, express or implied, or assumes any legal liability or responsibility for the accuracy, completeness, or usefulness of any information, apparatus, product, or process disclosed, or represents that its use would not infringe privately owned rights. Reference herein to any specific commercial product, process, or service by trade name, trademark, manufacturer, or otherwise, does not necessarily constitute or imply its endorsement, recommendation, or favoring by the United States Government or any agency thereof. The views and opinions of authors expressed herein do not necessarily state or reflect those of the United States Government or any agency thereof. 


\title{
DEMONSTRATION OF EMITTED-NEUTRON COMPUTED TOMOGRAPHY TO QUANTIFY NUCLEAR MATERIALS
}

\author{
P. A. Hausladen, M. A. Blackston, and J. Newby
}

Date Published: September 2011

Prepared by

OAK RIDGE NATIONAL LABORATORY

Oak Ridge, Tennessee 37831-6283

managed by

UT-BATTELLE, LLC

for the

U.S. DEPARTMENT OF ENERGY

under contract DE-AC05-00OR22725 



\section{CONTENTS}

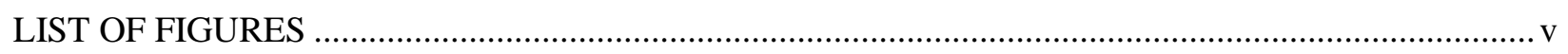

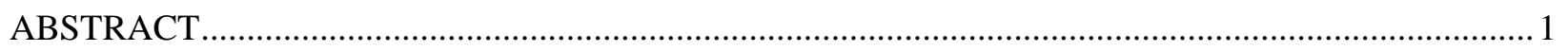

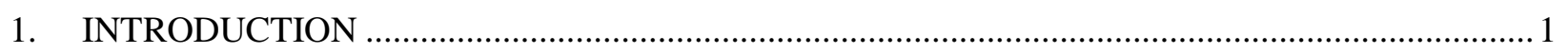

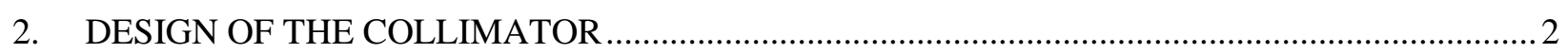

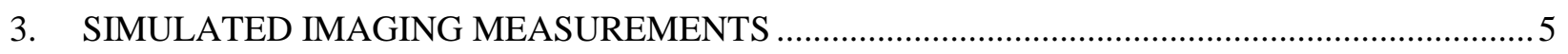

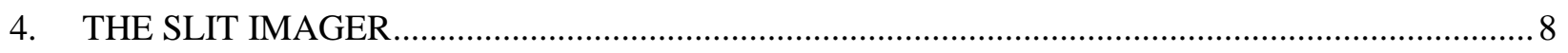

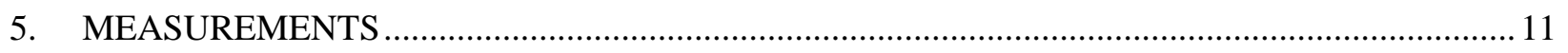

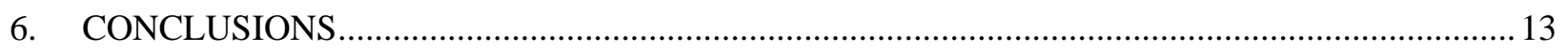

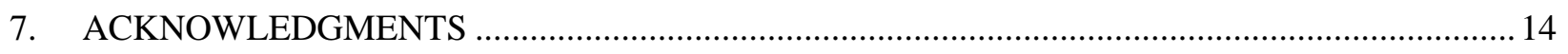

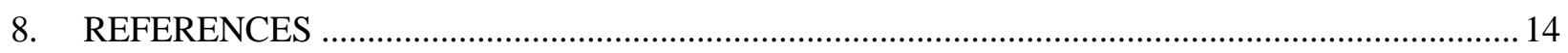





\section{LIST OF FIGURES}

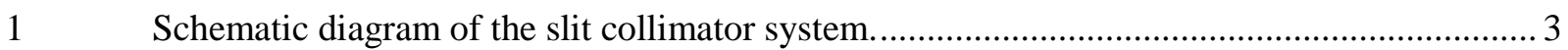

2 Response of the slit collimator to a point neutron source....................................................... 4

$3 \quad$ Results of slit collimator optimization simulations............................................................. 5

$4 \quad$ Results from a simulated measurement of a single point source............................................ 6

5 Comparison of simulated projections to the fitted results for a single point source.................. 6

$6 \quad$ Results from a simulated measurement of two point sources. .................................................. 7

$7 \quad$ Comparison of simulated projections to the fitted results for two point sources. ..................... 7

$8 \quad$ Photograph of a "soup can” containing an array of 32 Pu MOX rodlets. ............................... 8

$9 \quad$ Results from a simulated measurement of a soup can full of rodlets...................................... 8

$10 \quad$ Photographs of the slit-collimator imager.......................................................................

11 A pixelated liquid scintillator imaging detector................................................................ 9

12 Pixelated liquid-scintillator detector performance. ….......................................................... 10

13 Close-up photograph of radial collimator........................................................................... 11

14 Photographs of the fixture and sources used for measurements performed at ORNL........... 12

15 Results from a measurement performed at ORNL of five ${ }^{252} \mathrm{Cf}$ sources.............................. 12

16 Photograph of soup can \#4 from INL and resulting tomographic reconstruction.................. 13 



\begin{abstract}
In this document, we report demonstration of emitted-neutron computed tomography using fast fission neutrons to infer the geometry of sources of special nuclear material. The imaging system employed in the demonstration is based on a newly constructed array of pixelated neutron detectors suitable for arrangement in a close-packed imaging array and whose active volume consists of liquid scintillator EJ-309, which allows neutron-gamma discrimination via pulse shape to enable essentially pure fast-neutron imaging. The system is capable of high quality fast-neutron imaging where tomographic reconstruction of slices through an object resolves neutron sources similar in dimension to a fuel pellet, or about $1 \mathrm{~cm}$. During measurements of plutonium mixed oxide fuel rodlet arrays in soup cans at the Zero Power Physics Reactor facility at Idaho National Laboratory, the position of a partial defect of a single rodlet containing plutonium being replaced by one containing depleted uranium was detected.
\end{abstract}

\title{
1. INTRODUCTION
}

This report documents demonstration of emitted-neutron computed tomography using fast fission neutrons to infer the geometry of sources of special nuclear material (SNM). This work was performed under Fuel Cycle R\&D Material Protection and Control Technologies (MPACT) Campaign work package FTOR11MP0208, “Fast Neutron Imaging to Quantify Nuclear Materials.” In previous work, the authors demonstrated fast-neutron coded-aperture imaging using pixelated detectors developed at Oak Ridge National Laboratory (ORNL) and fabricated from plastic scintillator that cannot inherently distinguish neutron- and gamma-induced counts but whose sensitivity to gamma rays was minimized with lead shielding [1]. These measurements demonstrated that fast-neutron imaging was possible with suitable position resolution and a modest-sized device, but the results were not quantitative because of the unknown contribution from gamma rays. In addition, the signal-to-noise was limited by the large gamma-ray rate in the detectors. As a consequence, fast-neutron imaging detectors were developed whose active volume consists of liquid scintillator EJ-309, which allows neutron-gamma discrimination via pulse shape to enable pure neutron imaging [2]. The liquid-based detector design has the same outer and active volume dimensions as the previous plastic detectors and is suitable for arrangement in a close-packed array for imaging applications. An array of these liquid-based imaging detectors was built by a separate project and was available for use in the present work. With the availability of this fast-neutron imaging detector array, it was desirable to demonstrate the possibility of high-quality fast-neutron imaging where tomographic reconstruction of slices through an object was capable of resolving neutron sources similar in dimension to a fuel pellet, or about $1 \mathrm{~cm}$.

Fast-neutron imaging is appealing in scenarios where contact measurement with non-imaging detectors may be impractical or too labor intensive, or their results ambiguous because of surrounding material. For the present work, a representative problem that both served to demonstrate capability and could be investigated with the inventory of plutonium available at the Idaho National Laboratory's (INL's) Zero Power Physics Reactor (ZPPR) facility was needed. For this problem, the present work investigated tomographic imaging of fuel pins in an assembly by their neutron emanations in order to resolve individual pins as well as to detect replacement of a single pin with one not containing plutonium. This scenario could be investigated with a modest-sized imager that could be shipped to INL to image arrays of Pu mixed oxide (MOX) fuel rodlets arranged in the approved "soup can" geometry available at the ZPPR facility.

This report documents the design and simulated performance of the imaging system based on the number and form factor of the liquid scintillator detectors that were expected to be available, the construction of the imager, and its use in proof-of-concept tomographic imaging measurements of emitted fast neutrons 
from ${ }^{252} \mathrm{Cf}$ fission-neutron sources at Oak Ridge National Laboratory (ORNL) and Pu MOX fuel rodlet arrays in soup cans at the INL ZPPR facility. In the latter measurements, the replacement of a single rodlet containing Pu with one containing depleted uranium (DU) was detected. The success of these measurements marks a significant accomplishment. Since the detectors and acquisition were being developed in parallel by another project, no part of the imaging system had been used previously, and significant effort was required to have all of the components available and functioning for the measurements.

\section{DESIGN OF THE COLLIMATOR}

In general, fast-neutron imaging is difficult to perform for the very reason that it is desirable, namely, that fast neutrons penetrate a good deal of shielding. This property of fast neutrons allows them to escape from configurations of material that are highly attenuating to gamma rays. High-resolution imaging techniques are based on modulation of the incident flux by some sort of an aperture, the simplest of which is a pinhole. In the case of the pinhole, an image is formed on the detector plane when radiation incident on the pinhole from any position in the field of view can travel unhindered to the detectors, but radiation that is not incident on the pinhole is blocked. The resolution of the resulting image depends on the size of the pinhole, with a small pinhole necessary for good spatial resolution. Constructing a pinhole that is sufficiently small for good spatial resolution and sufficiently thick to modulate neutrons without attenuating neutrons that enter the pinhole from oblique angles represents the fundamental challenge of fast-neutron imaging. For an imaging system with a fixed focal length, this is possible by starting with the analog of a parallel-hole collimator, but pointing the individual holes toward a focus that acts as a virtual pinhole. Such a collimator is relatively easy to conceptualize but costly and complicated to build. However, because of the axial symmetry of an array of fuel rodlets in a soup can, the collimator can also have axial symmetry, reducing the pinhole imager to a slit imager. The slit imager is less complicated to build and at the same time has more open area in which to detect neutrons. Operationally, the use of a slit means that all the pixels from a single vertical column of pixels in the detector plane are summed.

A schematic diagram of the thick slit imager concept is shown in Figure 1. To the left of the diagram is the image plane made up of an array of previously developed pixelated fast-neutron detectors. Each of these detectors consists of a $10 \times 10$ array of approximately $1 \times 1 \times 5 \mathrm{~cm}^{3}$ optically isolated volumes of liquid scintillator. To the right of the detector array is the slit collimator. The slit collimator is made up of two components, an outer collimator labeled "thick pinhole" in Figure 1, and an inner collimator labeled "radial collimator." Typically, these collimators would be constructed of an excellent neutron attenuator such as high-density polyethylene (HDPE). The outer collimator serves to eliminate neutrons whose incidence is far from the slit, but it forms images with poor resolution because neutrons whose incidence is near the pinhole are not attenuated strongly. The inner or radial collimator serves to eliminate neutrons that would otherwise degrade the resolution because their paths were inconsistent with passing through the slit. This collimator will also eliminate some of the desirable neutrons; for instance, if the radial collimator obscures half of the area of the detectors, it will also eliminate half of the neutrons that passed through the slit. For the collimator in the example, the object to be inspected (such as a soup can) is placed to the right of the slit at a distance that is a third the distance from the slit to the detectors. This placement magnifies the image of the emitted radiation in order to gain higher resolution for the object, in this case by a factor of three. 


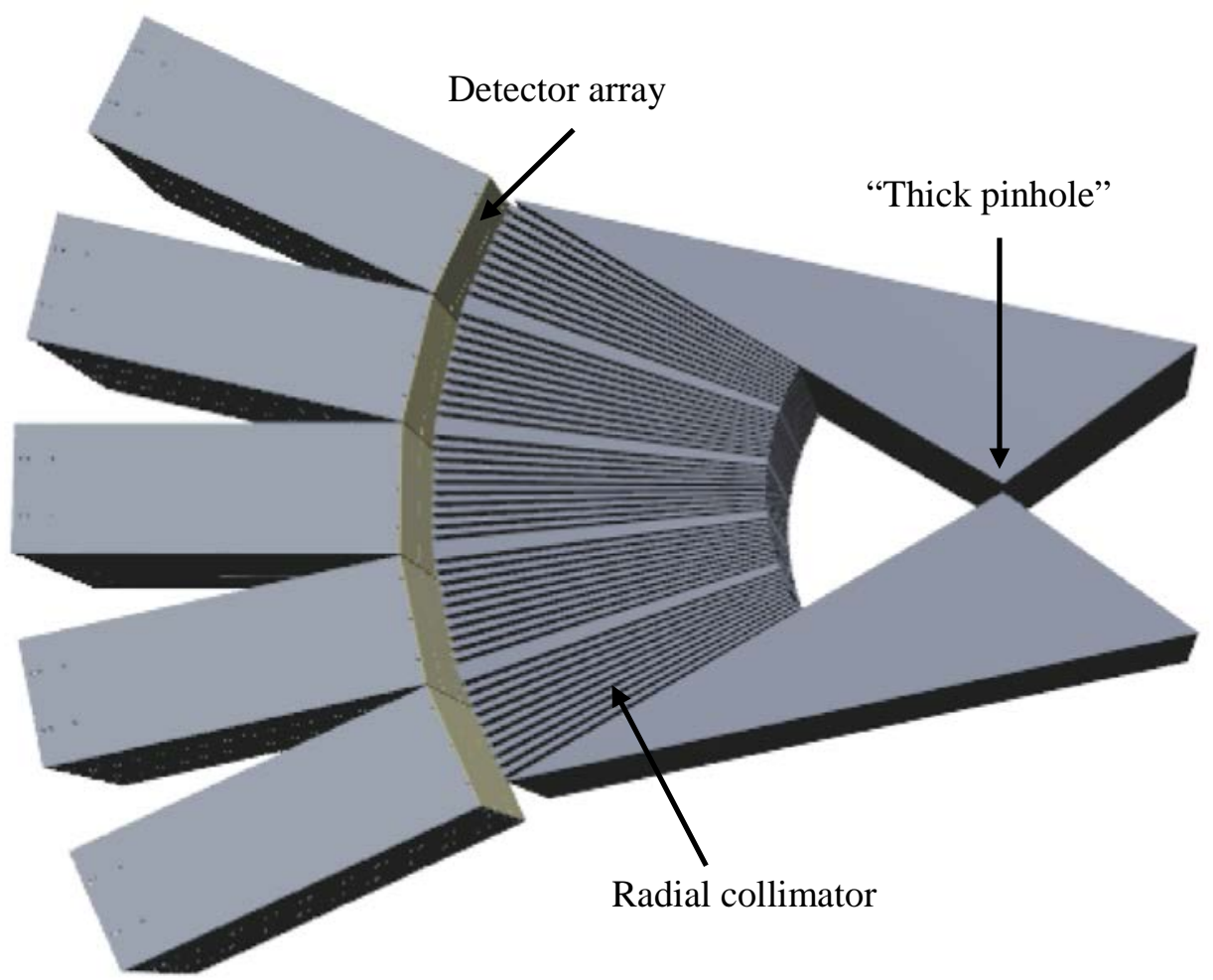

Figure 1. Schematic diagram of the slit collimator system. The system includes an image plane composed of pixelated detectors, a slit collimator here labeled "thick pinhole," and a radial collimator.

To illustrate the effects of the inner and outer collimators, the response of the slit collimator to a point neutron source at the center of the field of view was calculated using the GEANT4 Monte-Carlo simulation toolkit [3] and is shown in Figure 2. As indicated earlier, the outer collimator or "thick pinhole" has poor resolution but works well to eliminate counts at large pixel displacement. In contrast, the radial collimator has good resolution but does not eliminate counts at large pixel displacement. The combination of both components has both reasonable resolution and extinguishes counts at large pixel displacements. The composite response can be thought of as a Gaussian peak but with long exponential tails on either side. The imaging signal originates mostly from the Gaussian component, while the exponential tails mainly contribute noise to complicated images having more than a few neutron sources. 


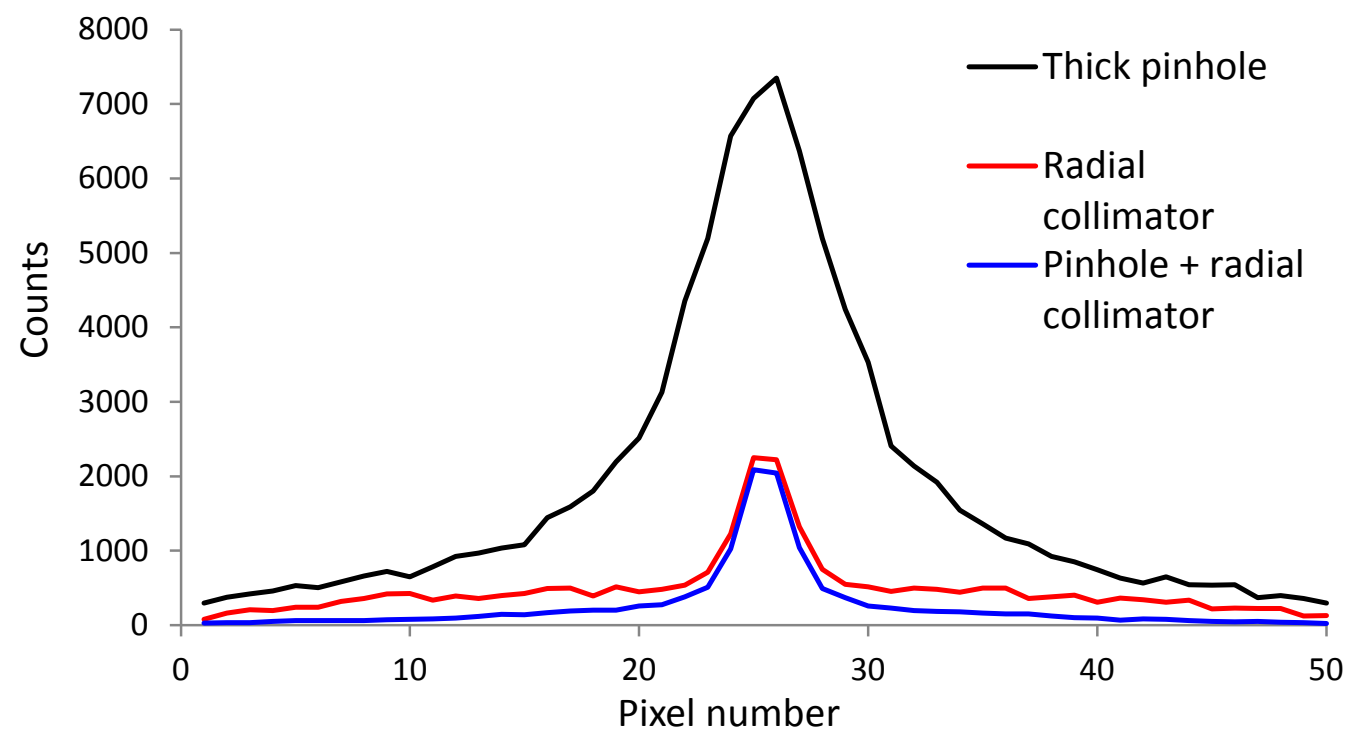

Figure 2. Response of the slit collimator to a point neutron source. The poor-resolution response of the outer collimator alone is labeled "thick pinhole." Likewise, the response of the inner collimator showing poor contrast at large pixel displacements is labeled "radial collimator."

With a basic understanding of the contribution of the different collimator components, simulations of a number of potential designs were performed in order to identify an optimal design. Given that the number and form factor of the detectors were predetermined by the availability of the imaging detector array, the collimator design was fully determined by a few parameters, namely, the width of the outer collimator opening (or slit width), the thick pinhole-to-detector distance (or slit-to-detector distance), and the open fraction of the radial collimator. Allowing these parameters to span reasonable values, more than a hundred configurations were simulated in order to identify the optimized design in terms of signal-to-noise and resolution. The results of these simulations are summarized in Figure 3. On the right side of Figure 3, it can be seen that the width of the higher-resolution Gaussian-like portion of the neutron response varies little for an open fraction less than about 0.5 , but the resolution rapidly deteriorates with open fractions greater than 0.5 . As a consequence, an open fraction of 0.5 was chosen. Note that geometries having a root mean square resolution of $\sim 1$ pixel (indicated by the dashed line) at the detector will be able to resolve sources separated by approximately $1 \mathrm{~cm}$ in the image when the positioning of the object gives a magnification of 3. On the left side of Figure 3, the signal-to-noise is shown as a function of both the slit width and the slit-to-detector distance. The red circle highlights a weak maximum in signal-to-noise in the vicinity of $8 \mathrm{~mm}$ slit width and $50 \mathrm{~cm}$ slit-to-detector distance. Consequently, a slit width of $8 \mathrm{~mm}$ and a $52.5 \mathrm{~cm}$ slit-to-detector distance were chosen. This slit dimension gives a nominal efficiency for neutrons from a source located $16.7 \mathrm{~cm}$ from the slit of $1.5 \times 10^{-4}$. 

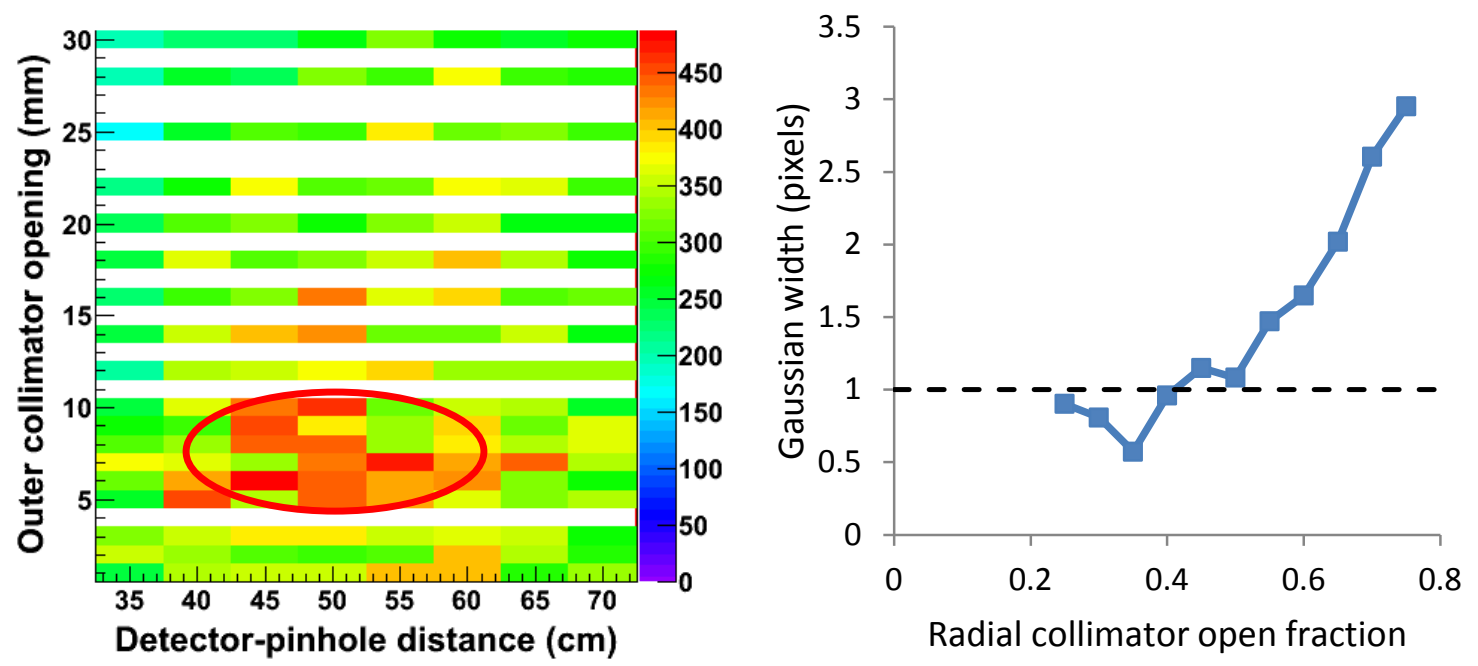

Figure 3. Results of slit collimator optimization simulations. (Left) The outer collimator opening and pinhole-detector distance were varied to optimize the figure of merit (FOM), which was defined to be the square of the Gaussian component of the signal divided by the total number of counts in the detector plane. The color scale represents the value of the FOM, and the red circle highlights the weak maximum in the FOM. (Right) The radial collimator open fraction was varied to optimize the width of the point-spread function. For the collimator that was constructed, an outer collimator slit of $8 \mathrm{~mm}$, a $52.5 \mathrm{~cm}$ slit-to-detector distance, and a $50 \%$ open fraction were chosen.

\section{SIMULATED IMAGING MEASUREMENTS}

The design-optimization simulations concentrated largely on calculating the "point spread function," or response across the detector array to a point source at the center of the field of view. In addition to these design optimization simulations, simulations were performed of tomographic imaging measurements where one or more sources was assumed to be on a rotational stage whose axis was centered in the field of view. A series of measurements were simulated at different rotational angles in order to enable tomographic reconstruction of a horizontal slice through the object. These simulations were used to develop and troubleshoot image reconstructions that take into account the calculated point spread function of the detector, to predict the expected imaging performance of the actual system when performing tomographic reconstruction, and to identify the expected level of statistical accuracy required to resolve a number of discrete neutron sources in the field of view.

To clearly demonstrate how the tomographic imaging works, it is easiest to first consider simulation of a tomographic measurement of a single point source. For this simulation, a point source offset from the center of rotation by $4 \mathrm{~cm}$ was measured at 36 different angles, each offset from the previous by $10^{\circ}$. The simulations corresponded to $3 \times 10^{6}$ neutrons per angle, equivalent to a source of 5000 neutrons s ${ }^{-1}$ for 10 minutes. For each angle, the pattern of counts on the detectors was simulated and recorded. These count patterns were summed by columns of pixels and were collected as a function of simulated measurement angle into a "sinogram," which is shown on the left of Figure 4 and used as input to the image reconstruction. The image reconstruction uses maximum-likelihood expectation maximization (MLEM), which iterates toward the best solution by calculating the expected count pattern for each angle from a trial distribution of neutron source, comparing it to the measured sinogram, at then updating the trial distribution. For the simulated point source, the trial source distribution after 30 iterations corresponds to the point source shown on the right of Figure 4, and the reconstructed or fitted sinogram is 
shown in the center. Good agreement between the reconstructed sinogram and the simulated measurement was obtained.
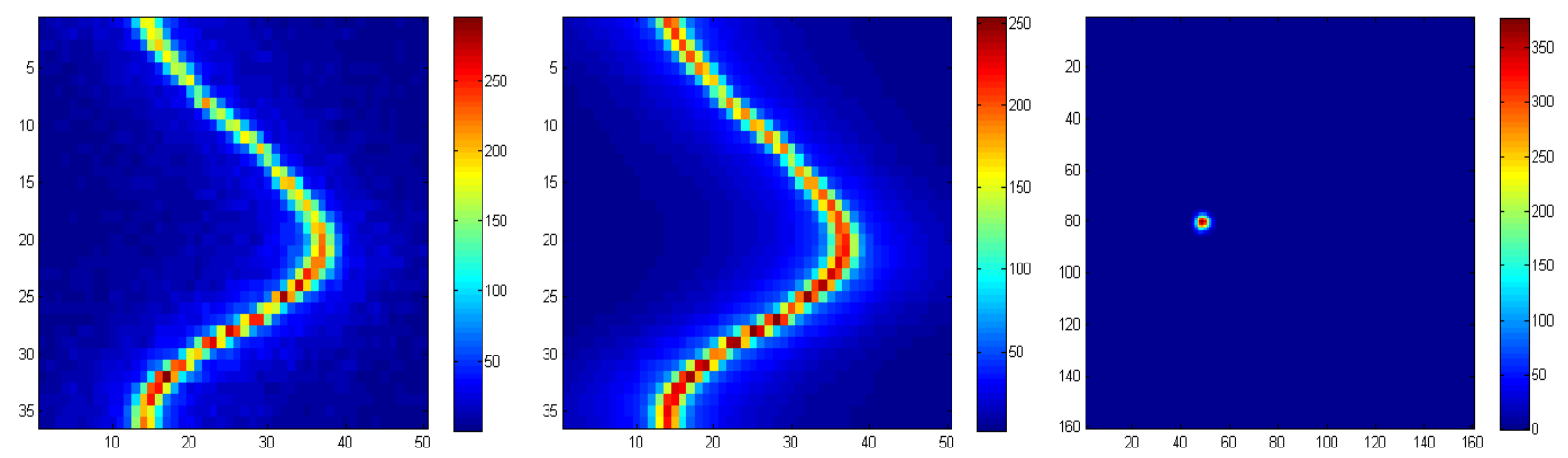

Figure 4. Results from a simulated measurement of a single point source. The plots correspond to the (left) simulated sinogram; (center) reconstructed, or fitted, sinogram; and (right) reconstructed image. In the sinograms, each row (or projection) corresponds to the hit pattern on the detector when the imaged object has been rotated to a given angle. From one row to the next, the object has been rotated by $10^{\circ}$.

While agreement appears very good between the simulated measurement and the reconstruction, it is often difficult to assess when judging solely from the color scale. As such, the simulated response (points) and MLEM reconstruction are shown for the $2^{\text {nd }}$ (black) and the $20^{\text {th }}$ (red) projections in Figure 5. It can be seen that the reconstruction captures most of the features of the simulated measurement, including the exponential tailing away from the central peak.

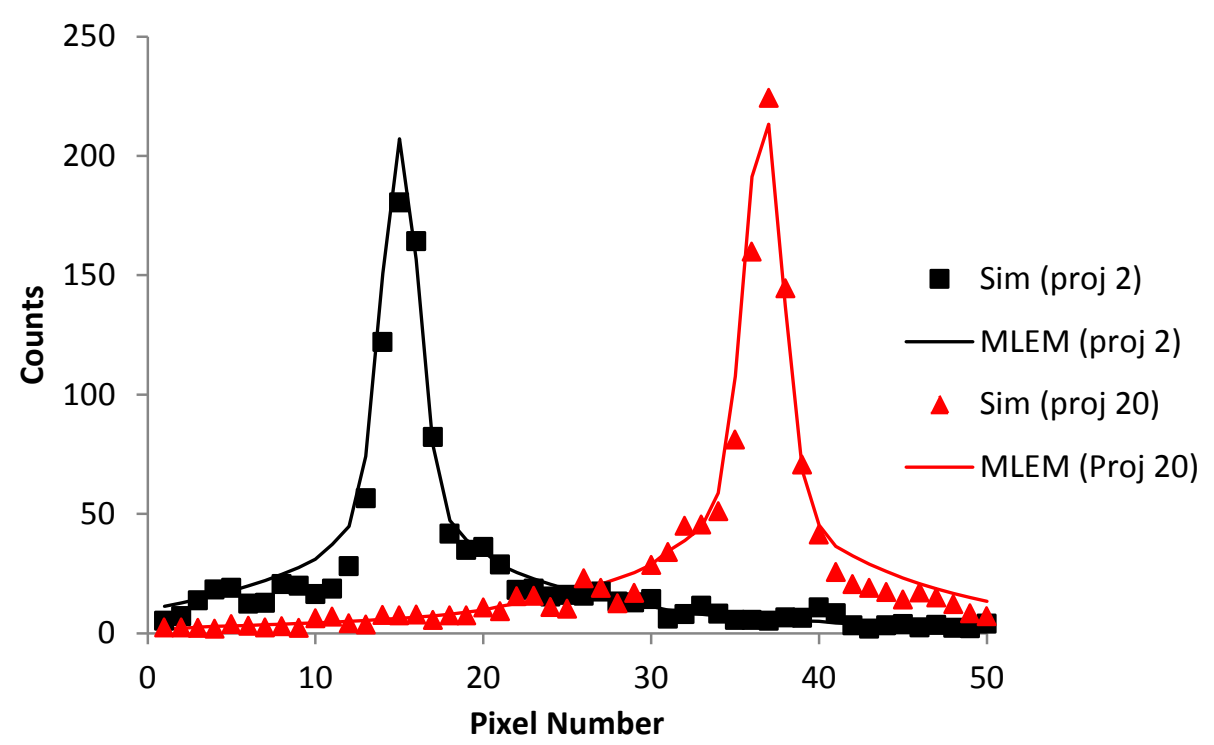

Figure 5. Comparison of simulated projections to the fitted results for a single point source. The simulated projections are indicated by points, and MLEM reconstructions are indicated by lines.

It is also useful to consider a pair of point sources to investigate how easily they can be resolved from each other. As such, simulations were performed of two point sources separated by $2 \mathrm{~cm}$. As in the case of the single point source simulations, 36 angles of the turntable were simulated. For each of these angles the simulated number of neutrons for each source corresponded to $3 \times 10^{6}$ neutrons emitted into $4 \pi$ 
steradians. Figure 6 shows the simulated sinogram for the two point sources, the reconstructed sinogram, and the reconstructed image following 30 iterations. Note that point sources separated by $2 \mathrm{~cm}$ are easily distinguished in the image. The simulated and reconstructed count distributions across the detector pixels are shown for two of the projections in Figure 7. Once again, agreement is good, even in the tails of the distributions.
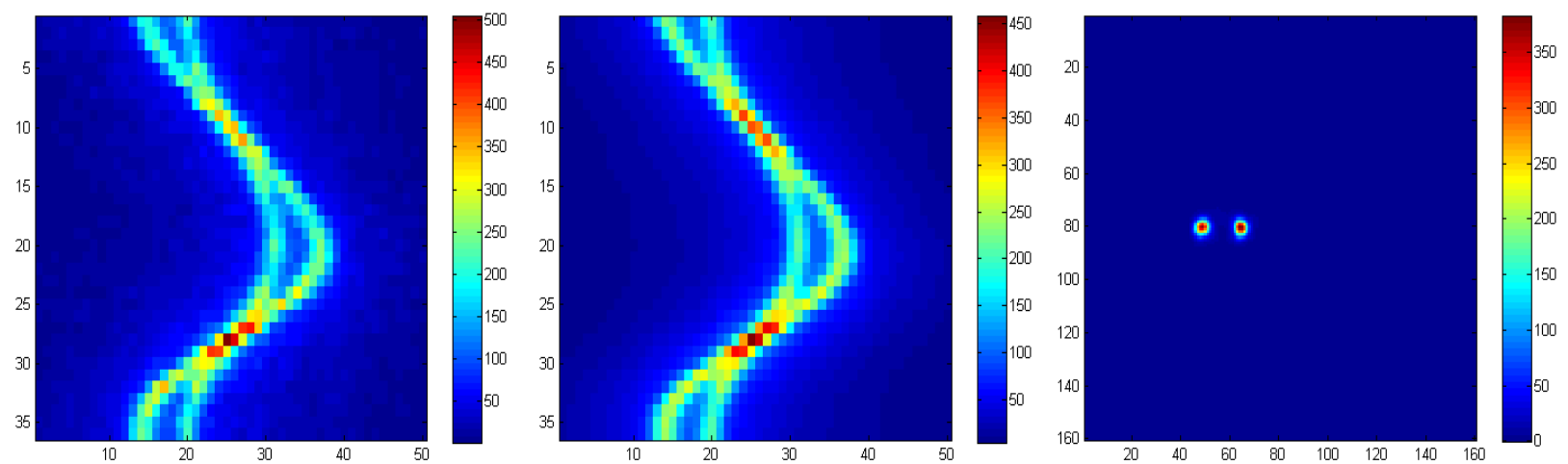

Figure 6. Results from a simulated measurement of two point sources. The plots correspond to the (left) simulated sinogram; (center) reconstructed, or fitted, sinogram; and (right) reconstructed image.

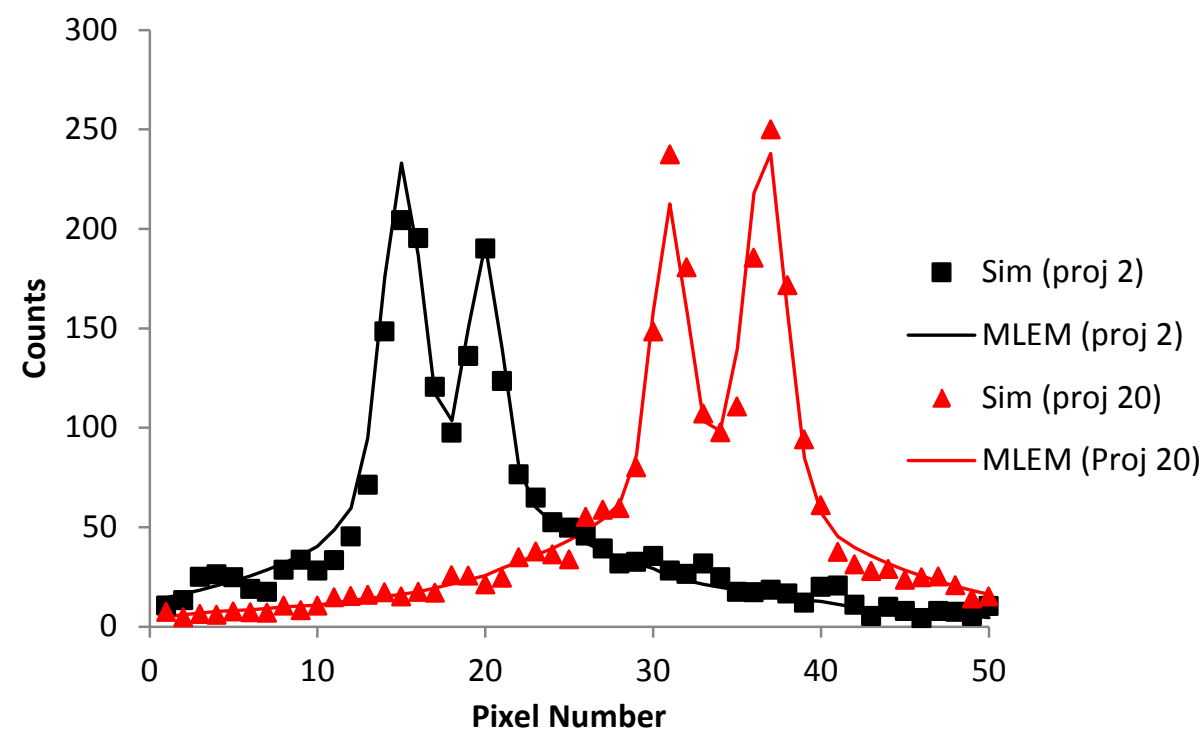

Figure 7. Comparison of simulated projections to the fitted results for two point sources. The simulated projections are indicated by points, and MLEM reconstructions are indicated by lines.

Following reconstructions of one or several point sources, simulations of arrays of point sources were performed. These simulations were intended to predict the capability of the imager for counting fuel rodlets in an array, such as could be populated at the INL ZPPR facility. For illustrative purposes, a photograph of an example array of $32 \mathrm{Pu}$ MOX fuel rodlets held in a soup can is shown in Figure 8. The rodlets are spaced from each other by half the rod diameter, similar to the packing of fuel rods in a fuel assembly. 


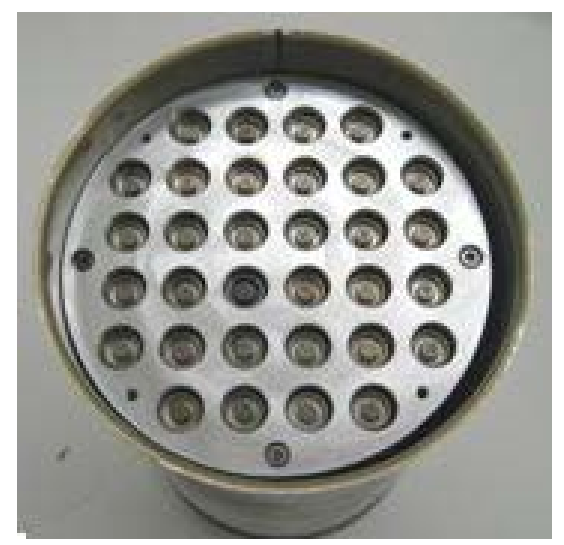

Figure 8. Photograph of a "soup can” containing an array of $32 \mathrm{Pu}$ MOX rodlets. Each rodlet is separated from its neighbor by half the rod diameter.

For the scenario of an array of rodlets in a soup can, the number of simulated neutrons was $3 \times 10^{5}$ neutrons per rodlet per projection, which corresponds to a source strength of 5000 neutrons $\mathrm{s}^{-1}$ for each rodlet and 1 minute of counting at each of 36 angles. This source strength corresponds to the actual source strength in neutrons per second of fuel Type 129 available at the ZPPR facility [4]. The simulated measurement can be seen in the sinogram in Figure 9 along with the reconstructed sinogram and the reconstructed image.
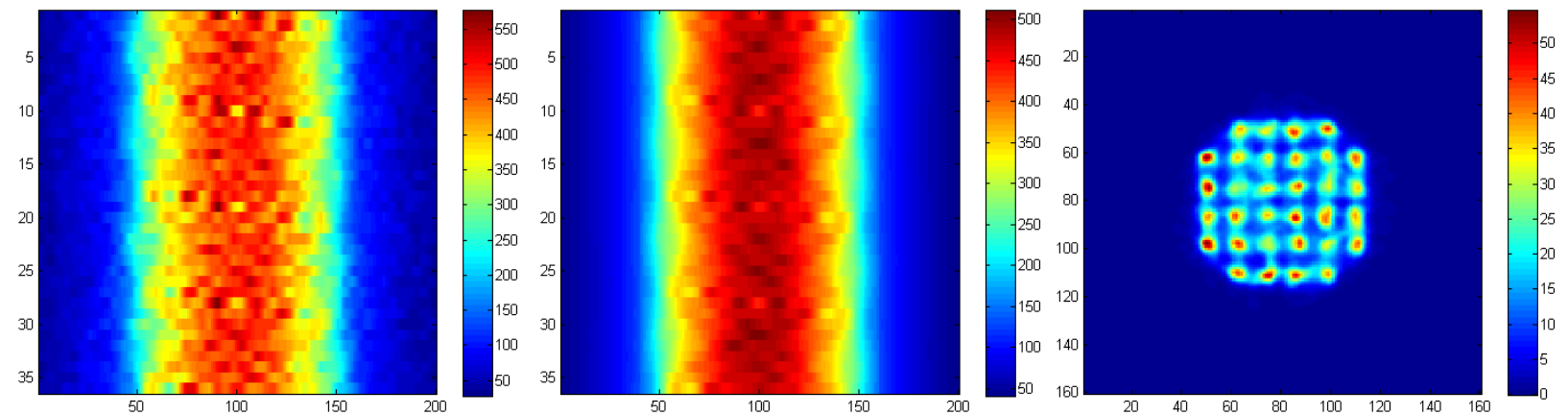

Figure 9. Results from a simulated measurement of a soup can full of rodlets. The plots correspond to the (left) simulated sinogram; (center) reconstructed, or fitted, sinogram; and (right) reconstructed image.

In this estimate, a 36 minute measurement is sufficient to count the individual fuel elements. However, this estimate does not include a background count rate in the detectors, the inevitable systematic measurement errors, or attenuation or scattering in the fuel elements. The combination of these factors can lengthen the actual measurement times significantly.

\section{THE SLIT IMAGER}

The slit imager was designed and built based on the parameters identified in simulations. The imager consisted of an image plane of pixelated detectors with corresponding data acquisition, the slit collimator, a turntable onto which soup cans or other neutron sources can be placed, and a D-T generator mounted on an arm that can sweep across the field of view to perform transmission imaging. Photographs of the assembled imager can be seen in Figure 10. The image plane is composed of 15 pixelated liquid scintillator 
imaging detectors arranged in five columns of three detectors each positioned along an arc that placed the detector front faces $52.5 \mathrm{~cm}$ from the slit position. In the photograph in Figure 10, only 10 of 15 of the detectors are installed. Photographs of an individual pixelated detector are shown in Figure 11, for a fully assembled detector, the same detector with the mu-metal shield removed to show the four-PMT readout and scintillator expansion chamber, and the pixelated scintillator chamber prior to assembly into the detector. Because these detectors are also sensitive to gamma rays, there is $2.5 \mathrm{~cm}$ of lead between the detectors and the slit collimator to shield the detectors from the abundant gamma rays emitted from Pu MOX fuel. Elastic scattering of neutrons from a heavy nucleus such as lead is forward peaked, so placement of the lead immediately in front of the detectors does little to affect the resolution of the system.
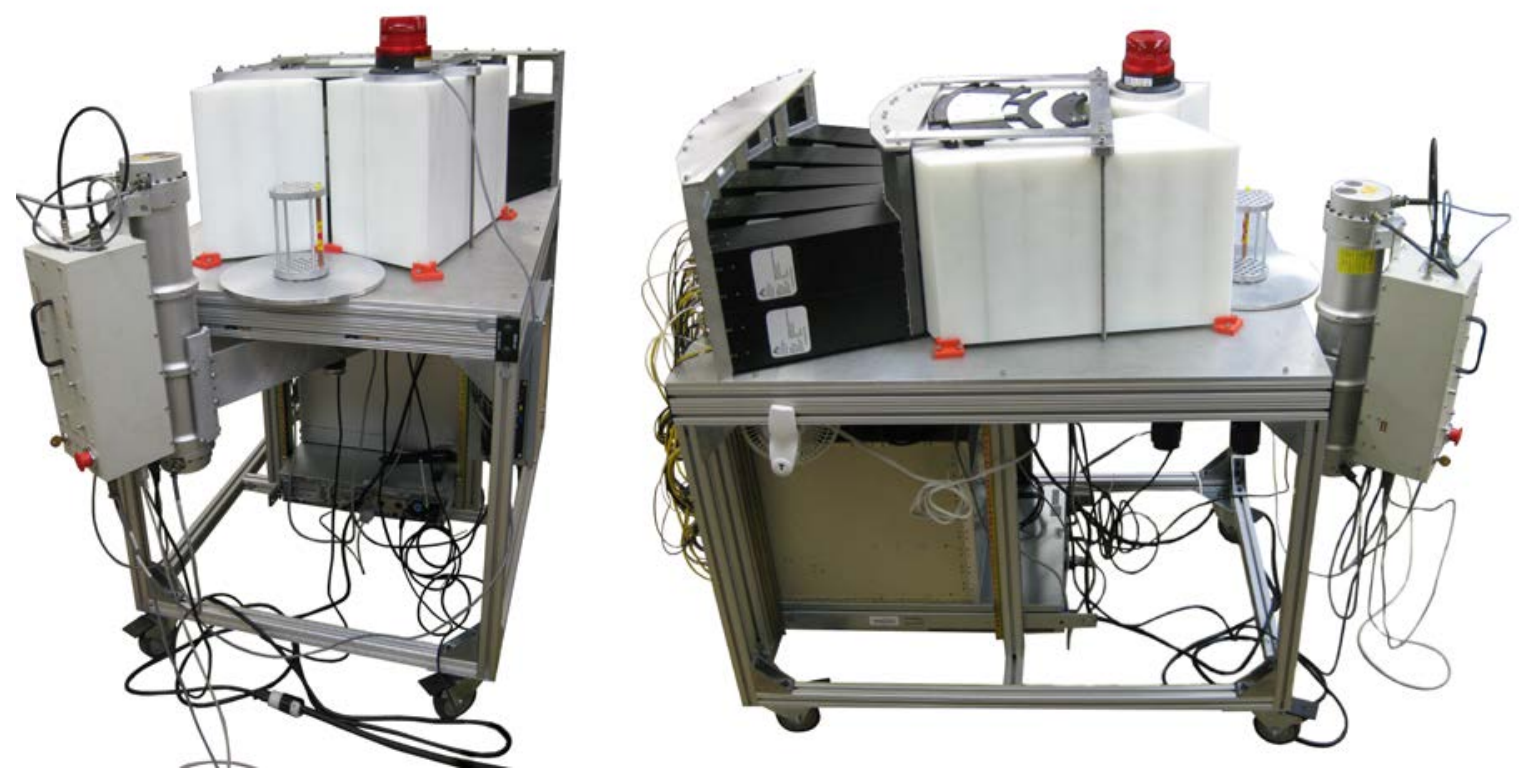

Figure 10. Photographs of the slit-collimator imager. Photographs provide (left) a view from behind the target object and D-T generator, and (right) a view from the side.
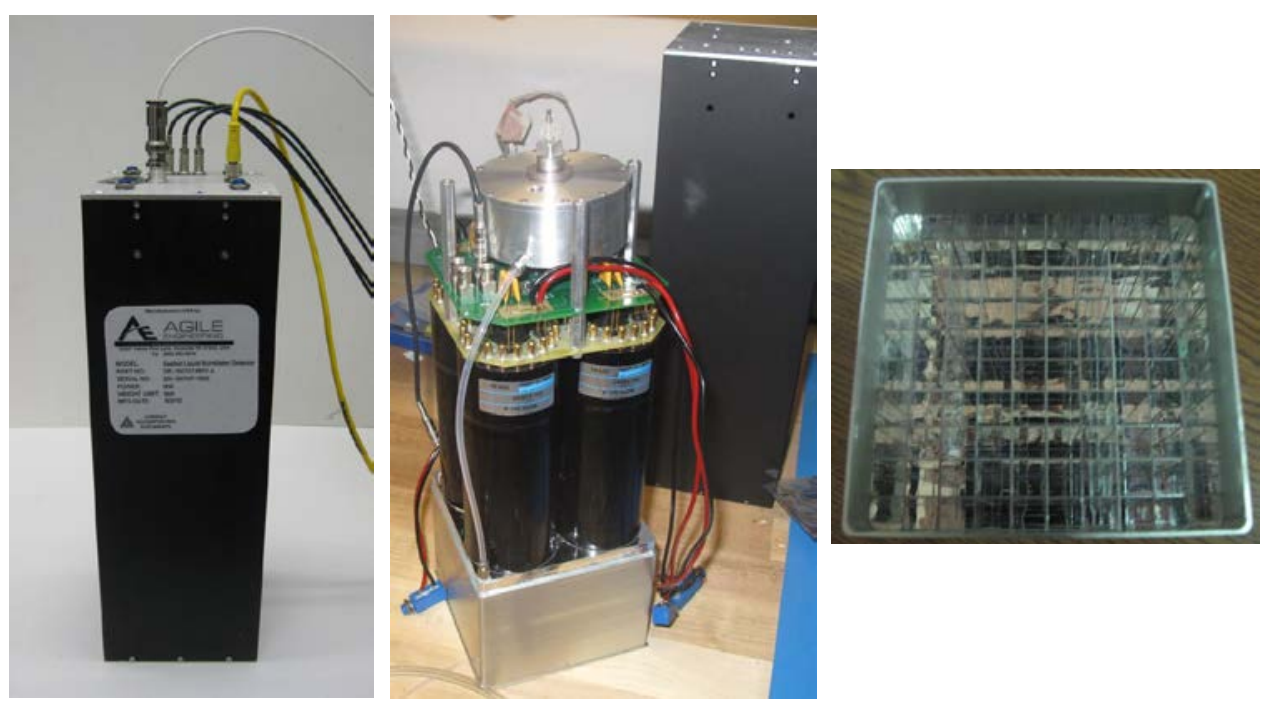

Figure 11. A pixelated liquid scintillator imaging detector. The detector is shown (left) fully assembled, (center) with the mu-metal shield removed to reveal the four PMTs and the scintillator expansion chamber, and (right) prior to assembly with the interior of the pixelated scintillator volume visible. 
Compared to previous coded-aperture imaging measurements with plastic-based detectors, use of the pixelated liquid-based detectors required a new data acquisition system that could record pulse-shape information. The new data acquisition consisted of four XIA Pixie-16 sixteen channel free-running $100 \mathrm{MS} / \mathrm{s}$ digitizers. The sum of four digitized waveforms (corresponding to the four PMTs of an imaging detector) was formed within an onboard processor to provide triggering with pulse-height independent timing via a trapezoidal filter. For each triggered event, a number of user-selected gates along all four waveforms was recorded so that the position could be reconstructed properly for interaction positions near the sides or corners of the detector where little light was detected. The positions of these gates were optimized for neutron-gamma discrimination. The measured pulse-shape and position response of a typical detector during calibration with neutrons from a D-T neutron generator is shown in Figure 12.
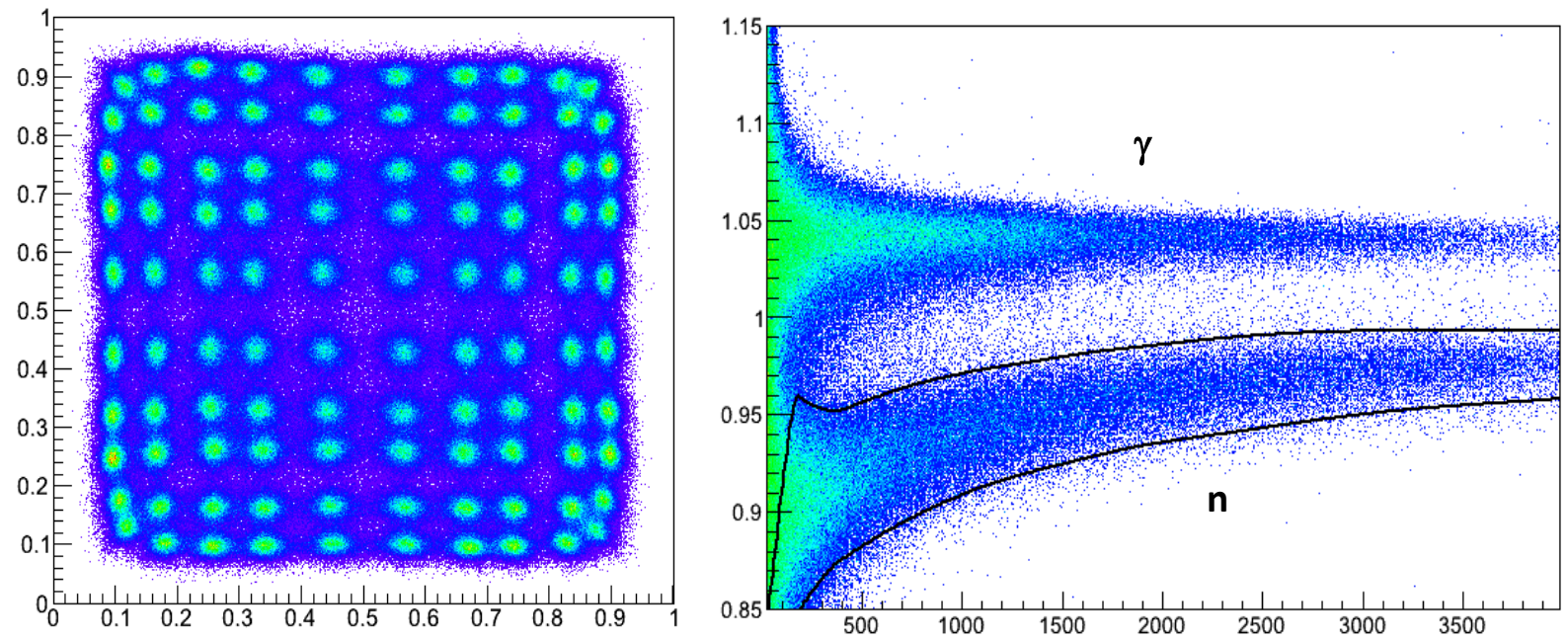

Figure 12. Pixelated liquid-scintillator detector performance. (Left) the position response of a detector with neutron selection is shown for uniform illumination by a D-T generator neutron source, and (right) the pulse-shape response as a function of pulse height is shown for the same source. Neutron events are selected within the outlined region on the pulse shape vs. pulse height plot.

The external slit collimator consisted of two large HDPE pieces. To ensure accurate location of the inside edges of the slit, the corners of the collimator were located by a set of brackets that were printed on a rapid-prototyping system that gave highly accurate inside edges for accurate alignment. The radial collimator was constructed of a set of 45 thin HDPE wedges that separated neighboring columns of pixels in the imaging detector array. Each of these wedges was $33.4 \times 29.8 \mathrm{~cm}$ and tapered from $4.7 \mathrm{~mm}$ thick at the detector end to $1.9 \mathrm{~mm}$ thick at the slit end. There were also four thicker wedges that separated pixels in adjacent detectors. The set of 49 HDPE wedges was also held in place by a set of brackets purpose-printed on a rapid prototyping system. After machining, the thin wedges of HDPE were subject to warping. To address this problem, the mounting brackets had slots to hold a series of four thin-walled tubes between each pair of wedges that served to separate the wedges from each other. This method of locating the wedges worked well at the thicker end, but some warping at the thin end was not entirely controlled, and inconsistent spacing can be seen between wedges at the thin end of the collimator in the photograph in Figure 13. This inconsistent spacing reduces the contrast of the collimator somewhat; however, there was insufficient time to find an adequate remedy prior to use of the collimator at the INL ZPPR facility. 


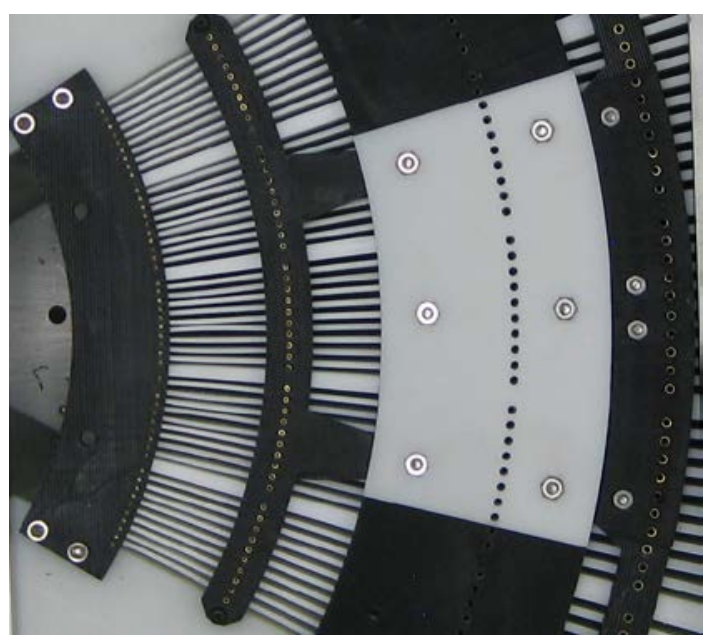

Figure 13. Close-up photograph of radial collimator. Bowing of the HDPE wedges at the thin end is visible.

\section{MEASUREMENTS}

The pixelated liquid scintillator detectors used in the present work were developed in parallel with the slit collimator by a separate project and were only completed in time to be available two weeks prior to shipment of the system for the scheduled measurements at INL. This late availability of the detectors entailed significant effort associated with use of largely untested equipment. Despite this late availability, proof-of-concept imaging measurements were performed with ${ }^{252} \mathrm{Cf}$ sources at ORNL prior to shipment of the equipment to INL, but the measurements were performed with only 10 of 15 detectors in place. For these proof-of-concept tomographic measurements, a set of five identical ${ }^{252} \mathrm{Cf}$ sources was used whose emission rates were each approximately 42,000 neutrons s${ }^{-1}$ as of February 2011. For the imaging measurements, each half-inch-diameter source was placed in a container formed by a piece of copper tubing with rubber stoppers in either end. The copper tubing has the same 3/8 inch outer diameter as a fuel rodlet, and the tubing containers holding sources were placed in five of the positions in a fixture identical to the ones used subsequently to hold the arrays of fuel rodlets at INL. The arrangement of the ${ }^{252} \mathrm{Cf}$ sources can be seen on the left side of Figure 14; the containers holding sources are labeled by yellow stoppers. The containers with black stoppers are holding tungsten carbide machining grit. For the imaging measurement, the source configuration was counted for 5 minutes, rotated $10^{\circ}$, and counted again until a total of 36 measurements were performed spanning $360^{\circ}$. In addition to the imaging measurement, the combined response of the detectors and slit collimator was characterized by a long measurement with these sources placed at the collimator slit. Calibration data such as this is also required to calibrate the position response of each detector and the pulse-shape response of each detector. The measured sinogram can be seen in Figure 15 along with the corresponding reconstructed sinogram after 30 iterations. The maximum likelihood distribution of neutron source corresponding to the reconstructed sinogram is also shown and clearly identifies the position of each neutron source. In fact, the two closest sources (whose centers are $1.5 \mathrm{~cm}$ apart) are easily resolved. 

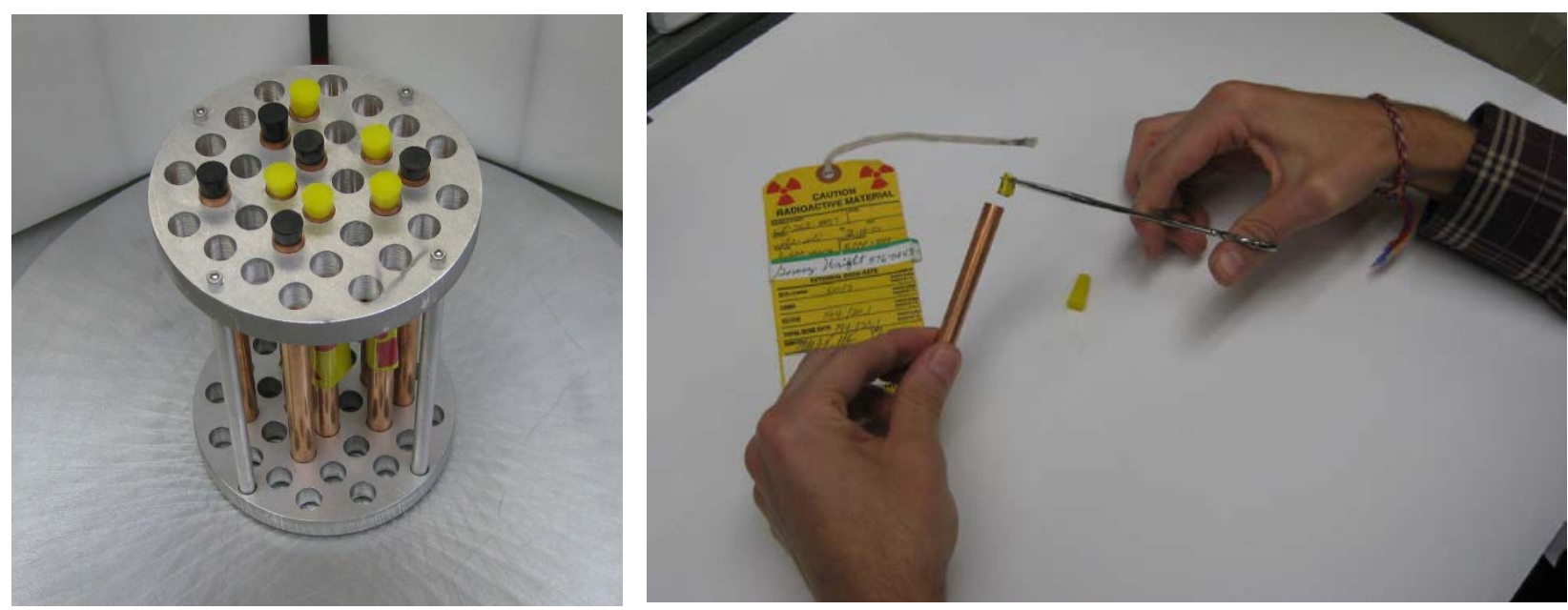

Figure 14. Photographs of the fixture and sources used for measurements performed at ORNL.

(Left) a fixture similar to that used at INL to hold fuel pins was used to hold ${ }^{252} \mathrm{Cf}$ sources. (Right) Tubing containing sources were labeled by yellow stoppers.
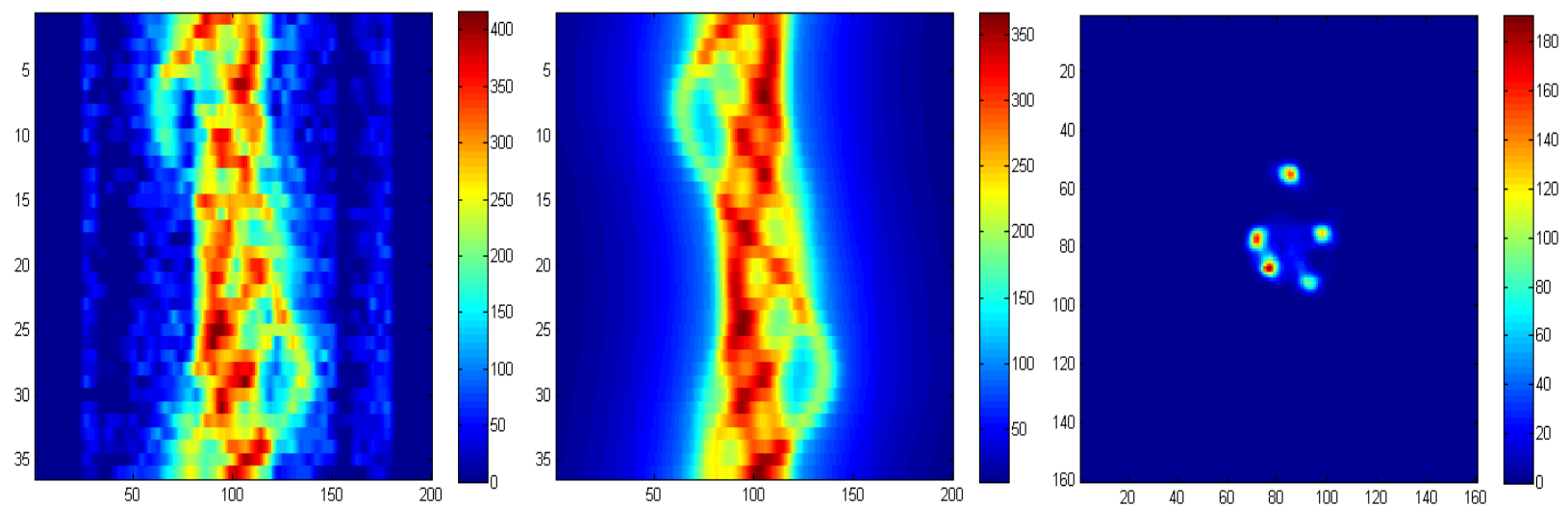

Figure 15. Results from a measurement performed at ORNL of five ${ }^{252} \mathrm{Cf}$ sources. The left plot corresponds to a measured sinogram showing changes to the hit pattern on the detector array as the source is rotated. For comparison, the (center) reconstructed sinogram can be seen along with the (right) reconstructed image which identifies the source configuration and resolves neutron sources whose centers are separated by $1.5 \mathrm{~cm}$.

Following a successful measurement at ORNL, the slit imager was shipped to the INL ZPPR facility. Unfortunately, the time available prior to shipment was insufficient to properly commission the transmission imaging capability. The transmission imaging was desirable both to be able to constrain the neutron emission sites during emitted-neutron image reconstruction to those where material is present and to correct for self-attenuation. However, the transmission imaging was only desirable if it could be performed much more quickly than the passive imaging. Because the transmission imaging was performed by sweeping the D-T generator across the field of view, only the small fraction of counts opposite the generator are used to measure transmission. As a consequence, to perform transmission imaging quickly, the data acquisition was required to operate at much higher rates than during passive imaging. In fact, it required higher data acquisition rates than were possible using the initial implementation of the data acquisition. At the ZPPR facility, sufficient time was spent on performing transmission imaging to identify that it would be too time consuming to perform during the present allotment of measurement time. 
As is often true, tomographic measurements of soup cans containing actual Pu MOX fuel proved more challenging than the simple-minded simulations indicated. For example, the amount of gamma-ray shielding on the sides and backs of the detectors was completely inadequate for measurement of Pu MOX fuel. One of the first tasks performed was to stack lead bricks to better shield the detectors. In addition, in the ZPPR workroom, the neutron background can be a significant fraction of the relatively small signal transmitting through the collimator, even when the source under inspection has an intrinsic rate of more than 100,000 neutrons $\mathrm{s}^{-1}$. High-quality measurements in such a setting require careful measurements of background and calibration of the detector-collimator combination. The combination of these factors led measurement times to be much longer than originally anticipated. Following this realization, a long measurement of "IO \#4" was performed. IO \#4 is pictured in Figure 16 and corresponds to a soup can having 32 positions for fuel pins, 31 of which are filled with 6 inch long by 3/8 inch diameter ID \#129 Pu MOX fuel rodlets each containing $3.66 \mathrm{~g}$ of ${ }^{240} \mathrm{Pu}$ that produces approximately 3700 neutrons s $\mathrm{s}^{-1}$ from spontaneous fission. The other actinide components of the fuel produce another approximately 1300 neutrons $\mathrm{s}^{-1}$ from alpha-n reactions. The remaining position in the soup can is filled by an ID \#130 DU fuel rodlet. This soup can was measured for 36 projections each for 10 minutes 45 seconds. The resulting tomographic reconstruction of neutron emission identifies the position of the missing pin.
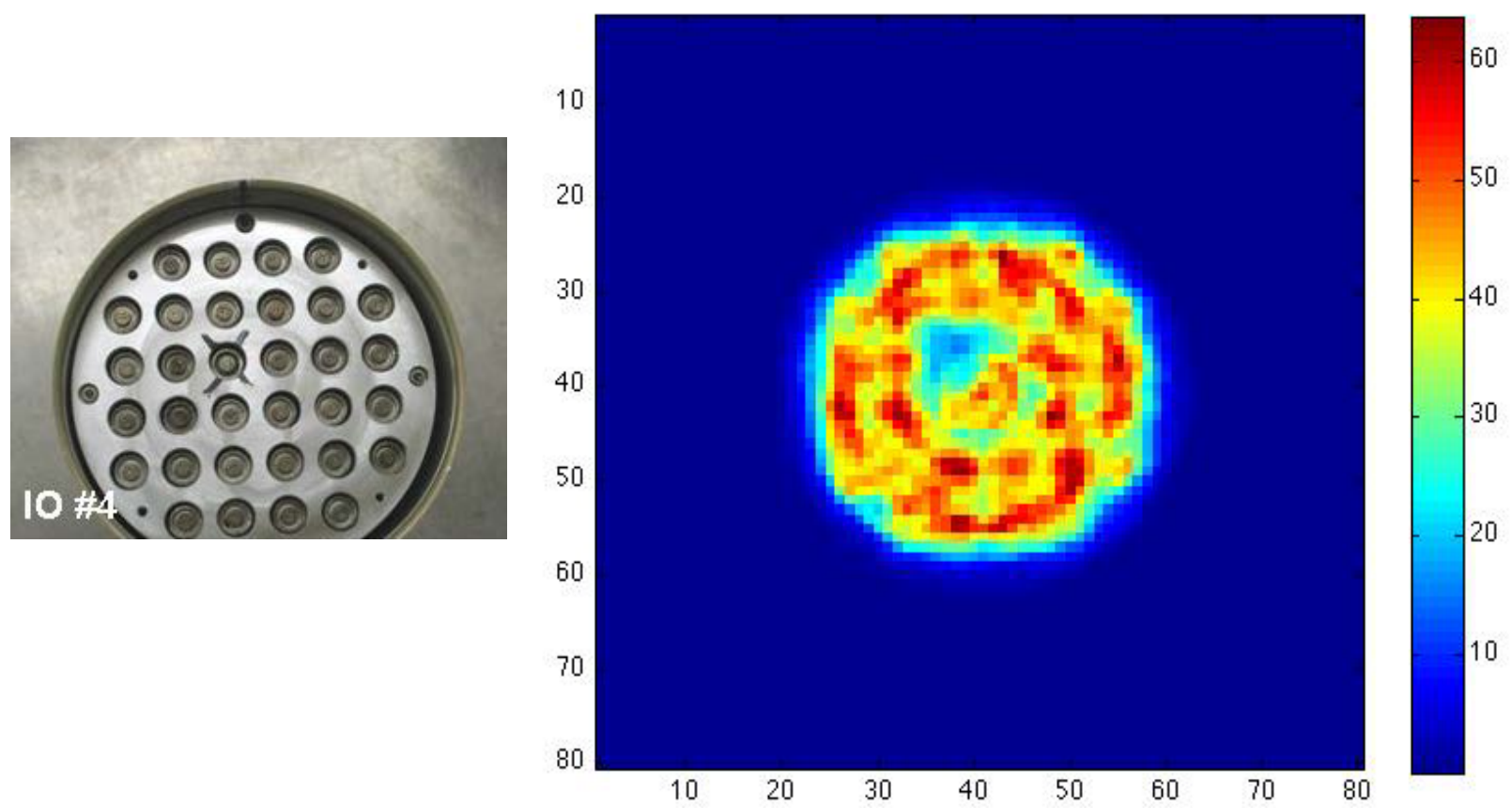

Figure 16. Photograph of soup can \#4 from INL and resulting tomographic reconstruction. (Left) The soup can holds 31 Pu MOX fuel pins and a single DU pin in the position marked by the "x." (Right) The tomographic reconstruction of neutron emissions identifies the position of the missing pin.

\section{CONCLUSIONS}

In the current year of this project, we have designed, constructed, and commissioned a fast neutron imager and successfully demonstrated emitted-neutron computed tomography using fast fission neutrons to infer the geometry of sources of special nuclear material. The design optimizations guided by simulated measurements were tested in simple, well-controlled laboratory experiments. Our first measurements at INL ZPPR successfully imaged the geometry of an assembly of plutonium fuel rodlets with one plutonium rodlet replaced by a depleted uranium rodlet, with the result that the replaced pin was 
identifiable. Furthermore, the measurement at the INL ZPPR facility provided an opportunity to identify problems associated with measuring with high-rate sources in realistic, high background environments. In future efforts, appropriate attention will be given to properly shielding the detectors to minimize the amount of radiation entering the detectors through paths around the collimator.

\section{ACKNOWLEDGMENTS}

The experiments described in this report were performed with collaborators from Idaho National Laboratory (INL) and Sandia National Laboratories (SNL). The authors gratefully acknowledge David Chichester and Scott Watson of INL for coordination and support prior to and during the measurements at the INL ZPPR facility, and Peter Marleau and Erik Brubaker of SNL for support with development of the data acquisition software and assistance with the INL measurements. The authors further acknowledge Robert Neibert and the ZPPR staff for proficient and resourceful assistance during unanticipated schedule conflicts.

\section{REFERENCES}

[1] P. A. Hausladen et al., ORNL/TM-2009/210.

[2] P. A. Hausladen et al., ORNL/TM-2010/201.

[3] J. Allison et al., IEEE Trans. Nucl. Sci. 53(1), 270-278 (February 2006).

[4] D. L. Chichester et al., INL/EXT-09-16566. 